\title{
PLOTTING EQUATIONS OF THREE VARIABLES IN MENTAL MEASUREMENTS
}

\author{
BY HERBERT A. TOOPS \\ Ohio State University
}

The possibility of using a series of curves, common and representative series values of a variable, to represent on ordinary plotting paper the variations of a dependant third variable of a mathematical equation, is not well known. The clearness with which these three-fold relationships can be visualized thereby, and the ease and accuracy with which such charts can be constructed for some of the simpler mathematical equations used by a clinical psychologist, would seem to recommend them to his use.

The two indices, commonly used by the clinician as comparative measures of mental ability, are cases in point. The first of these, the C.I.A. of the Yerkes-Bridges Point Scale, has the equation:

$$
\text { C.I.A. }=\frac{\text { Score made by subject }}{\text { Norm for age of subject }}
$$

And of the second, the I.Q. of the Terman or Stanford Revision of the Binet Scale:

$$
\text { I.Q. }=\frac{\text { Mental age of subject }}{\text { Chronological age }} \text {. }
$$

Each of these equations is the result of a simple division of variables of the first degree. It is quite possible to represent all the relationships of each of these equations, each made up of three variables, by means of a simple series of straight line curves, thus eliminating the use of clumsy tables, logarithms, and the like. Ordinarily we are interested in plotting only representative values (in this case, mostly used values) of one of the variables. The I.Q.'s, for instance, are never plotted for values of chronological age above 16 , nor below 3 . The principle used is that of proportionality of similar 
right triangles. Let us refer to the dotted lines of Fig. I. If on the ordinate-axis we lay off a series of decimal values, 0.00 to $\mathrm{r} .00$ inclusive, in any convenient scale, and then draw a line through the origin of the axes and intersecting the horizontal line which goes through the ordinate (1.00) at the point, $a$, we shall have the line, Oa. This line, Oa, serves to indicate the quotients of a whole continuous series of

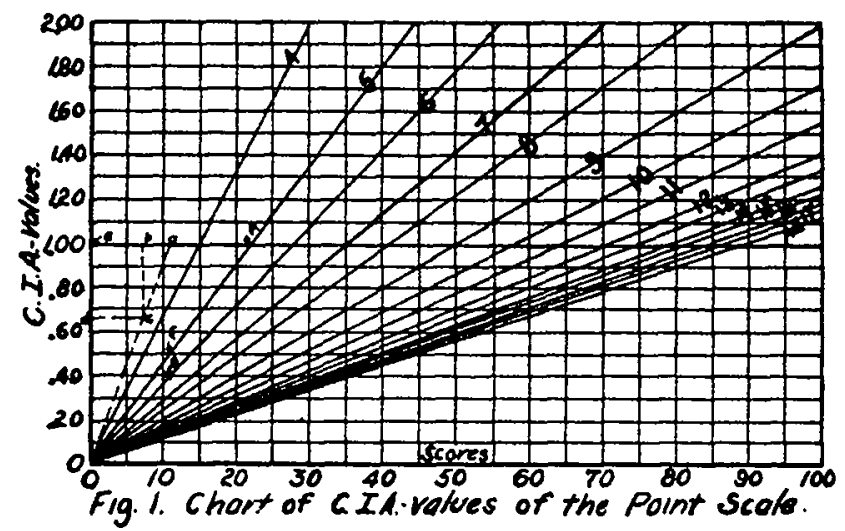

Fig. I. Chart of C. I. A. values of the Point Scale.

divisions of a variable by a constant quantity. It indicates the quotients obtained by dividing every possible value between $e$ and $a$ successively by the quantity, $e a$. If we consider any given abscissa value, $b e$, and from the point where the ordinate line of $b$ cuts the line, Oa, project across to the ordinate-axis we find the value, $O d$, which is the quotient of be/ae. The proof of this and the applicability to mental coefficients will now be shown.

Let $b$ be any point on the horizontal line through the ordinate, 1.00. Then, in the similar triangles, $a O_{e}$ and $c O d$, $O d / O e=c d / a e$, but since, $O e=1.00$, and $c d=b e$,

Therefore,

$$
O d=b e / a e \text {. }
$$

Or, $O d$ is the quotient of be divided by ae. This holds true for any point, $b$, irrespective of its position on the abscissaaxis and also irrespective of the scale in which either ordinates or abscissas are plotted. By extension of the line, Oa, the 
straight line curve serves to indicate quotients larger than unity for values of $b$ greater than $b e$ as divided by the value, ae. Multiplying the quotients thus obtained by any constant quantity-say by 100 as is commonly done for these mental coefficients - has no effect on the principle involved. We may label the quotients whatever we wish so long as the series of labels is obtained from the quotients of the diagram by multiplication by a constant quantity.

Applying this to our C.I.A. values: If we let the abscissas represent all possible successive scores on the Point Scale from 0 to 100 , and draw our line, $O A$, through the point whose abscissa is the norm for the age 5 and ordinate is 1.00 , then the line, $O A$, will serve to indicate all possible C.I.A. values that might be made by a child five years old for all the scores possible for him to make, within the limits of the chart. Accordingly we label this line a 5-year line. The norm for this age is 22, and a 5 -year-old who makes II points on this Scale (we see by tracing across the point, $f$, where the IIordinate cuts the line, $O A$ ), has a C.I.A. of .50. A 6-year-old who makes the same score, II, (we see by tracing across from g) has a C.I.A. of .39. In similar manner, each age line is drawn through the score (with C.I.A. of I.00) which is the norm for that age. ${ }^{1}$

The advantages of such a chart are many. It eliminates all computation and secures a distinct saving of time where quotients are needed quickly or where one has many to compute. The scale of the chart may be made large enough to secure any desired degree of accuracy. It can be constructed with the aid merely of pencil and straight edge. If the norms should be changed, the addition of an age line at the proper new norm would allow for the change, a thing not possible with a table of computed values. The interrelations of the coefficients are easily grasped. Ever recurring questions, such as, "What score would it have been necessary for the subject to make in order to have secured such-and-such-a coefficient" are answered by a glance at the

1 Yerkes, R. M., and Wood, L., 'Methods of Expressing Results of Measurements of Intelligence,' Jour. of Educ. Psych., Vol. VII., No. 10, 1916, pp. 593-606. 
chart. We see also why there is so much more variation of C.I.A. values in the lower ages than in the higher ages; for, one point difference in score makes but a difference of little more than one point difference (O.OI) in C.I.A. at the age 16 , while at the age 4 it makes a difference in C.I.A. of almost 7 points $(0.07)$. This is a great fault of the C.I.A. values. The almost horizontal angle of the higher age curves and the almost vertical angle of the lower age curves make for opposite extremes in their effect upon the C.I.A. values.

A chart constructed in like manner for I.Q. values has already been published." Similar charts used for expressing the quotient of one variable of the first degree divided by another variable also of the first degree, which quotient may then be multiplied by any constant quantity, may as easily be constructed for all such equations.

A further extension of this principle may be made to other simple mathematical equations often used in the psychological clinic. Two examples will suffice to illustrate the application.

Let us consider the Spearman formula for rank correlation,

$$
\rho=\mathrm{x}-\frac{6 \Sigma D^{2}}{n\left(n^{2}-1\right)} .
$$

This may also be written,

$$
\rho=\mathrm{I}-\frac{6}{n^{3}-n} \cdot \Sigma D^{2}
$$

Now, $\Sigma D^{2}$ has a maximum value when there is a perfect inverse correlation (when $\rho=-\mathfrak{x}$ ), or when

$$
\mathbf{I}-\left(\frac{6}{n^{3}}-n\right) \cdot \Sigma D^{2}=-\mathbf{I} \text {. }
$$

That is (solving for $\Sigma D^{2}$ ), when

$$
\Sigma D^{2}=\frac{n^{3}-n}{3} \text {. }
$$

The expression (6) is then an expression of the greatest possible deviation for all values of $n$.

'Toops, H. A., and Pintner, R., "A Chart for the Determination of I. Q. Values," Journal of Delinquency, Vol. III, No. 6, 1918, p. 272 + chart. 
For any given single value of $n$, the expression, $\left[6 /\left(n^{8}-n\right)\right]$ is a constant quantity and with $\Sigma D^{2}$ values calculated, equation (5) is a straight line function which may be plotted on the coördinates-ordinates $(\rho)$ and abscissas $\left(\Sigma D^{2}\right)$. There will, of course, be a straight line curve for each value of $n$. In

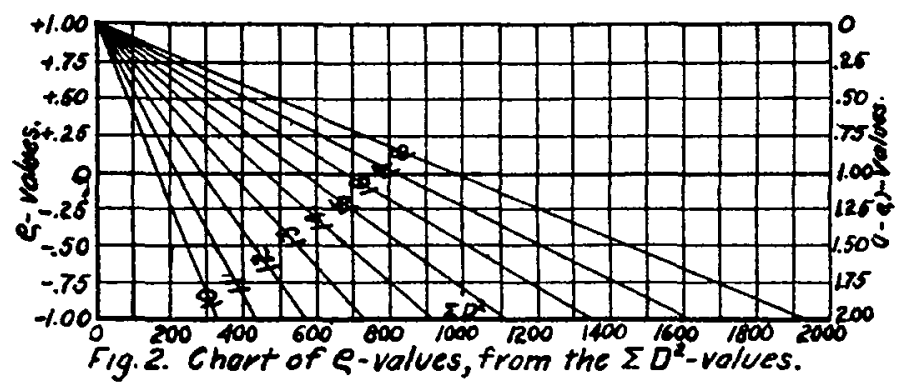

Fig. 2. Chart of $\rho$-values, from the $\Sigma D$-values.

Fig. 2 we have plotted several of these values. Any other curve for any particular value of $n$ desired may be drawn with a ruler and pencil, taking care only that the line pass through the nodal point $(\rho=+1.00)$, and through the maximum $\Sigma D^{2}$ on the line $(\rho=-1)$, through the $\left[\left(n^{3}-n\right) / 3\right]$

\section{TABLE I.}

Maximum $\Sigma D^{2}$-values Corresponding to Values of $n$.

\begin{tabular}{c|c|c|c|c|c|c|c}
\hline$n$ & Max. $\Sigma D^{n}$ & $n$ & Max. $\Sigma D^{n}$ & $n$ & Max. $2 D^{n}$ & $n$ & Max. $\Sigma D^{n}$ \\
\hline 9 & 240 & 23 & 4,048 & 37 & 16,872 & 51 & 44,200 \\
10 & 330 & 24 & 4,600 & 38 & 18,278 & 52 & 46,852 \\
11 & 440 & 25 & 5,200 & 39 & 19,760 & 53 & 49,608 \\
12 & 572 & 26 & 5,850 & 40 & 21,320 & 54 & 52,470 \\
13 & 728 & 27 & 6,552 & 41 & 22,960 & 55 & 55,440 \\
14 & 910 & 28 & 7,308 & 42 & 24,682 & 56 & 58,520 \\
15 & 1,120 & 29 & 8,120 & 43 & 26,488 & 57 & 61,712 \\
16 & 1,360 & 30 & 8,990 & 44 & 28,380 & 58 & 65,052 \\
17 & 1,632 & 31 & 9,920 & 45 & 30,360 & 59 & 68,440 \\
18 & 1,938 & 32 & 10,912 & 46 & 32,430 & 60 & 71,980 \\
19 & 2,280 & 33 & 11,968 & 47 & 34,592 & 61 & 75,640 \\
20 & 2,660 & 34 & 13,090 & 48 & 36,848 & 62 & 79,422 \\
21 & 3,080 & 35 & 14,280 & 49 & 39,200 & 63 & 83,328 \\
22 & 3,542 & 36 & 15,540 & 50 & 41,650 & 64 & 87,360 \\
\hline
\end{tabular}

value for the $n$ considered. The maximum $\Sigma D^{2}$ values for plotting the curves with other values of $n$ are given in Table I.

We often desire to know just what is the effect upon the correlation of an apparently aberrant case. This may be 
easily found, within certain definite limits to which it may be confined, from Fig. 2, as may be seen from the following considerations: Equation (5) may also be written,

$$
(\mathbf{x}-\rho)=\left(\frac{6}{n^{3}-n}\right) \cdot\left(\Sigma D^{2}\right) \text {. }
$$

These $(\mathrm{I}-\rho)$ values may be used as ordinates with exactly the same set of curves as for equation (5). Any particular deviation in rank, $h$, will give to its own particular deviation of the formula a value, $D^{2}=h^{2}$. It will, however, affect the other rankings also, as necessarily some of these are displaced by the aberrant ranking. The maximum effect of this displacement is when the lowest rank of ranking $A$ becomes the highest rank of ranking $B$, or vice versa. Ordinarily, however, only part of the ranks are displaced by the aberrant rank; if the aberrant rank is shifted down, all those displaced measures above the aberrant measure are each shifted one point in rank, and vice versa. The maximum effect, when one extreme measurement of one ranking becomes the other extreme of the other, is thus represented by the expression,

$$
D^{2}=h^{2}+(n-\mathrm{I}) \cdot(\mathrm{I})=\left(h^{2}+n-\mathrm{I}\right) .
$$

On a purely chance proposition, the average value of $(n-I)$ of the above equation would be $(n-1) / 2$; for practical purposes with $n$ very large, the effect of $(n-1)$ may be disregarded.

We can best grasp the meaning of equation (8) by assuming that the correlation of any two series of measurements would be perfect if it were not for the deviations. Each deviation serves to reduce the correlation by an amount corresponding to a $(1-\rho)$ value for its representation in $\Sigma D^{2}$, which must be at least $\left(h^{2}\right)$ and not more than $\left(h^{2}+n\right.$ - I). The $\rho$-limits corresponding to these may be found on Fig. 2 by taking the $(I-\rho)$ value (righthand ordinates) corresponding to $\left(h^{2}\right)$ and to $\left(h^{2}+n-1\right)$ taken as $\Sigma D^{2}$ values (abscissas). Aside from its theoretical interest, such a chart is especially valuable when one has a number of trial correlations to be calculated by this method. 
Another more specialized application of the principle is shown by the equation of the P.E. of the above $\rho$-values.

$$
\text { P.E. }=(0.706) \cdot \frac{\left(1-\rho^{2}\right)}{\sqrt{n}} .
$$

Assuming that to be valid or acceptable, a correlation, $\rho$, must be $a$ times the P.E. $\rho$, we may construct a series of line curves to show the number of cases necessary with a given $\rho$ to secure a greater reliability, or ratio of $\rho /$ P.E. $\rho$.

Our assumed measure of reliability of $\rho$ is:

$$
a=\frac{\rho}{\text { P.E.p }} .
$$

Then, in order to be valid, the P.E.p must be no more than the value, $\rho / a$, or

$$
\text { P.E. } \rho=\frac{\rho}{a}=(0.706) \cdot\left(\frac{1-\rho^{2}}{\sqrt{n}}\right) .
$$

Solving for $n, \rho \sqrt{n}=(0.706) \cdot(a) \cdot\left(\mathrm{I}-\rho^{2}\right)$

$$
n=(0.498) \cdot(a)^{2} \cdot\left[\left(\mathrm{I}-\rho^{2}\right) / \rho\right]^{2} .
$$

For any particular value of $a$, the expression $\left(0.498 a^{2}\right)$ is a constant quantity. Also the expression $\left[\left(1-\rho^{2}\right) / \rho\right]^{2}$ may be plotted as abscissas of a simple linear series of values and the appropriate $\rho$-values to which they refer (not an arithmetical series when plotted) so labeled. The straight line curves of Fig. 3 are labeled from the $a$-value from which they are derived, and not for the actual values used in plotting them. They can easily be plotted from the value of $n$ when $\left[\left(1-\rho^{2}\right) / \rho\right]^{2}$ is taken to be 100 in case of each value of $a$ taken. Then, by our principle already given, with the straight line through the origin of the axes and the point,

$$
\left[\left(\frac{\mathrm{I}-\rho^{2}}{\rho}\right)^{2}=100 ; n=n_{100}\right] \text {. }
$$

the value of $n_{100 / 4}$ must be $\mathrm{I} / A \cdot n_{100}$. Or, taking a concrete example, $n_{50}=1 / 2 \cdot n_{100}$. The expression $\left[\left(1=\rho^{2}\right) / \rho\right]^{2}$ is thus treated always as the independent variable, and as though 
it were a simple variable, $x$, so far as plotting is concerned. An example will best illustrate the use of Fig. 3 .

With a correlation, $\rho=0.4$, we find by tracing across to the left the point, $h$, where the $(\rho=0.4)$-ordinate cuts the

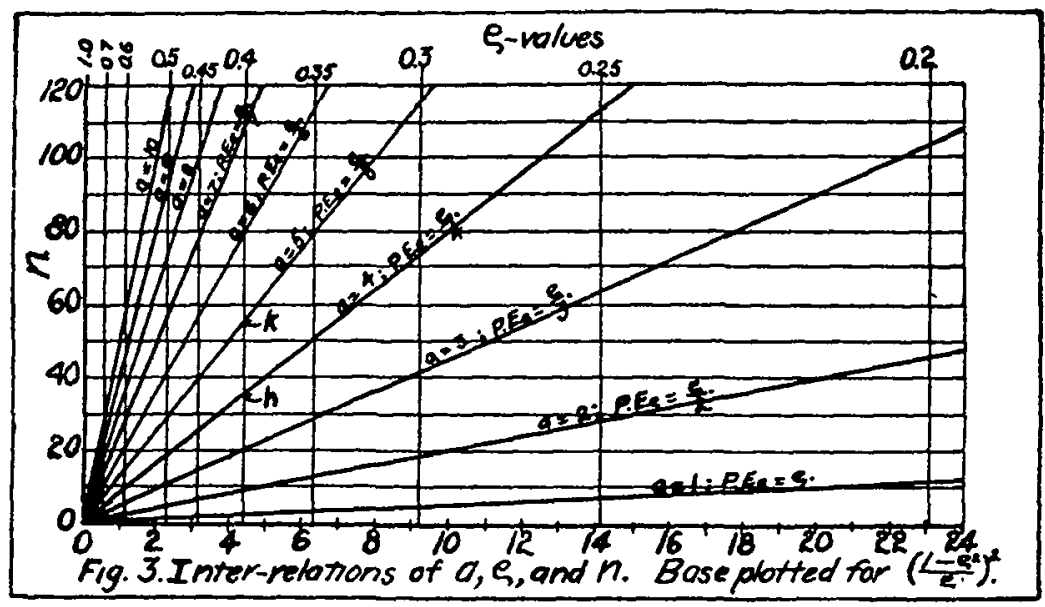

FIG. 3. Interrelations of $a, \rho$, and $n$. Base plotted for $\left[\left(1-\rho^{2}\right) / \rho\right]^{2}$. With a correlation $\rho=0.4$, we find by tracing across to the left the point, $h$, that 35 cases are necessary in order that the P. E. $\rho$ shall be only one fourth as large as $\rho$. In order to reduce the ratio so that the P. E. $\rho$ shall be only $1 / s$ of $\rho$, we project across the point, $k$, and find that 55 cases will be necessary.

( $a=4$ )-curve that 35 cases will be needed in order that the P.E. $\rho$ shall be only one fourth as large as $\rho$. Since the P.E. $\rho$ $=\rho / a$, in this case it is $0.4 / 4=0 . \mathrm{I}$. Now, if we wish to know how many cases we need with the same correlation (0.4) to secure an $a$-value of 5 , we have but to project the point, $k$, across to the left, whereupon we find that 55 cases will be necessary. The P.E.p here is 0.08 .

By this procedure we have reduced an equation of three variables and of the second degree to a chart of straight line curves.

In like manner we may plot any equation of the general formula, $y=A \cdot Z^{v} \cdot X^{w}$, in which $A$ is a constant quantity, $Z^{*}$ is a variable of any degree of which commonly used and representative curve values are desired, $X^{w}$ is either a complicated or simple expression which is to be treated in plotting 
as a simple variable, $x$. When $X^{w}$ is plotted along the lower base line of our plotting paper, we may then plot along the upper base line of the plotting paper as a geometrical variable a series of values (which may be computed for intermediate values), corresponding to the linear or arithmetical series below, the variations of the variable expression (not necessarily $x$ as shown above) which produces $X^{*}$. The individual curves are then to be labeled from the $(Z)^{1}$ value from which they were obtained. In all cases, the variations should be examined to see whether they may profitably be plotted as straight line curves. In general, it is unprofitable if $w$ is larger than 3 , unless only small ranges are taken of the variable expression which produces $X^{*}$; $v$ should also preferably be small or unity.

Fig. 4 illustrates a method of plotting $\left(y=a \cdot x^{2}\right)$ in such manner that not only common and representative values of

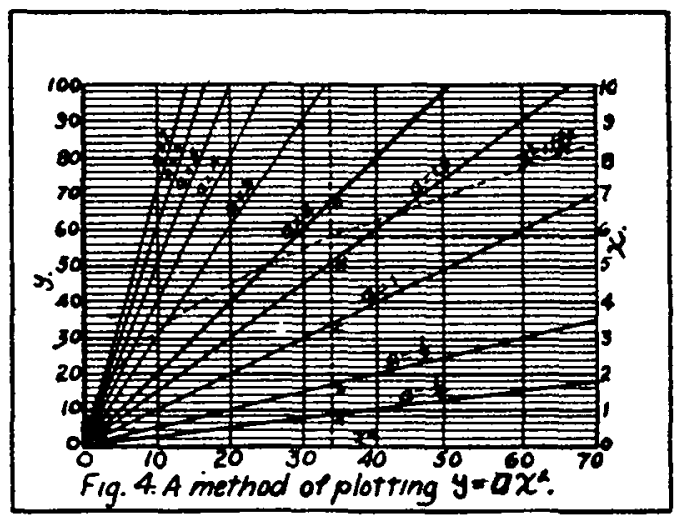

Fic. 4. A method of plotting $y=a x^{2}$. An $x$-value of 5.8 , on the right-hand scale, traced across to the left until it hits the parabola and then projected downwards to the base gives us an $x^{2}$-value of 33.6. Projected upward until hitting the $(a=1 / s)$-line at $a$ and then projected to the left we secure a $y$-value of 18.4 . That is, for the equation, $y=1 / 4 x^{2}$; when $x$ is 5.8 , then $y$ is 18.4 .

$x$ may be used but also every possible value. This is secured by plotting the curve of $x^{2}=\mathrm{I} \cdot(x)^{2}$ in such manner that the curve represents the square of the $x$-values which are plotted on the right-hand border, and thus being able to project the $x^{2}$-values to the base line. From the dotted lines 
of the figure, we see that with an $x$-value of 5.8 we secure an $x^{2}$-value of 33.6 ; this ordinate intersects the $(a=1 / 4)$ straight line curve at the point, $a$, corresponding to a $y$-value, 8.4. This means that for the equation, $y=1 / 4 x^{2}$, when $x$ is 5.8, the value of $y$ is 8.4. From the intersection of this ordinate with the other curves at points, $b, c, d$, $e$, we can readily find the value of $y$ when the value of $a$ respectively is, $1 / 2,1,1.5$, and 2 . The accuracy of the plotting of the curve, $x^{2}=\mathrm{I} \cdot(x)^{2}$ can always be tested by comparing the $x^{2}$-values determined from it with the value of $y_{(a-1)}$ determined by the intersection, $c$; the line, $a=1$, being very carefully plotted. 